\title{
Aberrant Rotator Cuff Muscles: Coexistence of Triple-Tailed Teres Minor and Bi-Formed Infraspinatus (Major and Minor)
}

\author{
James Olumide Ashaolu1, Oladipo Olayemi Abimbola ${ }^{1}$, Victor Okoliko Ukwenya ${ }^{2}$, \\ Magnus Adebo Thomas ${ }^{1}$ \\ ${ }^{1}$ Department of Anatomy, Bowen University, Iwo, Nigeria \\ ${ }^{2}$ Department of Anatomy, Ekiti State University, Ado Ekiti, Nigeria \\ Email: olusco172003@yahoo.com
}

Received 3 November 2014; revised 16 December 2014; accepted 29 December 2014

Academic Editor: David Pérez Jorge, Department of Teaching and Educational and Behavioral Research, Universidad de La Laguna, Spain

Copyright (C) 2015 by authors and Scientific Research Publishing Inc.

This work is licensed under the Creative Commons Attribution International License (CC BY). http://creativecommons.org/licenses/by/4.0/

(c) (i) Open Access

\begin{abstract}
The teres minor and infraspinatus muscles bear a close anatomical relationship in the posterior aspect of the scapula and constitute part of the rotator cuff muscles. Although there are only occasional reports of variations of the rotator cuff muscles, this paper reports the first combined occurrence of triple-tailed teres minor and infraspinatus muscle possessing two distinct parts, infraspinatus major and minor which are of clinical and sporting importance. Aberrant variations of rotator cuff muscles need to be brought to the knowledge of radiologists, surgeons and anatomists.
\end{abstract}

\section{Keywords}

Rotator Cuff Injury, Teres Minor Muscle, Infraspinatus Muscle, Anatomical Variation

\section{Introduction}

The understanding of the variability of the rotator cuff tendon anatomy can help towards its accurate clinical diagnosis and repair in cases of injury. The rotator cuff muscles, namely, the supraspinatus, infraspinatus, teres minor and subscapularis, hold the head of the humerus in the small and shallow glenoid fossa of the scapula [1] [2]. They are important in shoulder movements and in maintaining glenohumeral joint stability [1]. The infraspinatus muscle originates from the infraspinous fossa, in the dorsal aspect of the scapula and inserts on the 
posterior facet of the greater trochanter of humerus [1]. It occasionally receives some fibres from the teres minor and has not been previously reported to display pronounced structural variations [2]. It functions specifically in the lateral rotation of the humerus, along with the teres minor [1] [2]. On the other hand, the teres minor emerges from the lateral border of the scapula and attaches with the inferior aspect of the greater trochanter of humerus [1] [2]. We currently report a new occurrence of a triple-tailed teres minor, having each of the tails terminating independently on the proximal part of the humerus and a bi-formed infraspinatus muscle. This kind of variation may prove difficult to classify either during surgical or radiological encounter.

\section{Case Report}

The observations described below were made coincidentally on a male cadaver possessing triple-tailed teres minor and bi-formed infraspinatus muscle during routine supervision of medical students' dissection classes in the Anatomy Department of the College of Health Sciences, Bowen University, Iwo, Osun State, Nigeria. No history of the cadaver was available. The cadaver was preserved in preservative fluid consisting of $10 \%$ formalin, glycerol, phenol and acetic acid. Photographic records were taken with a digital camera (Samsung S630, Olympus digital camera E-410, made in China) for permanent recording.

We found two muscles seated in the infraspinous fossa and we classified them as the infraspinatus major and the infraspinatus minor (Figure 1, Figure 2 and Figure 4). The infraspinatus major originates from the main and medial part of the infraspinous fossa. It is musculo-tendinous and its fibres converge onto a tendinous end after two-third of its course.

The tendinous end inserts on the superior facet of greater tubercle of humerus. The infraspinatus minor originates from the lateral aspect of infraspinous fossa and the superior aspect of the inferior angle of scapula. It is completely muscular throughout its course. Some of its medial fibres partially attaches with the infraspinatus major along its course. It inserts on the posterior facet of the greater tubercle of humerus. The teres minor lies infero-lateral to the fibres of the infraspinatus minor (Figures 1-4).

The origination of the teres minor is quite robust. The teres minor possesses three tails, namely; the superior, middle and inferior tail. The superior tail is the shortest and inserts abruptly at the posterior part of greater tubercle, the middle tail is distinctly tendinous and moves somewhat anteriorly and attaching to the inferior facet of greater tubercle of humerus (Figure 3 and Figure 4). The inferior tail is musculo-tendinous and moves more anteriorly, attaching to the antero-inferior aspect of greater tubercle of humerus. The supraspinatus inserts on the superior facet of the greater tubercle of humerus. Axillary nerve supplies the teres minor while infraspinatus major and minor are supplied by suprascapular nerve. An arterial branch from the posterior circumflex humeral artery passes laterally and on the belly of teres minor to enter the space between the superior and middle tails of teres minor (Figure 3). All findings are unilateral.

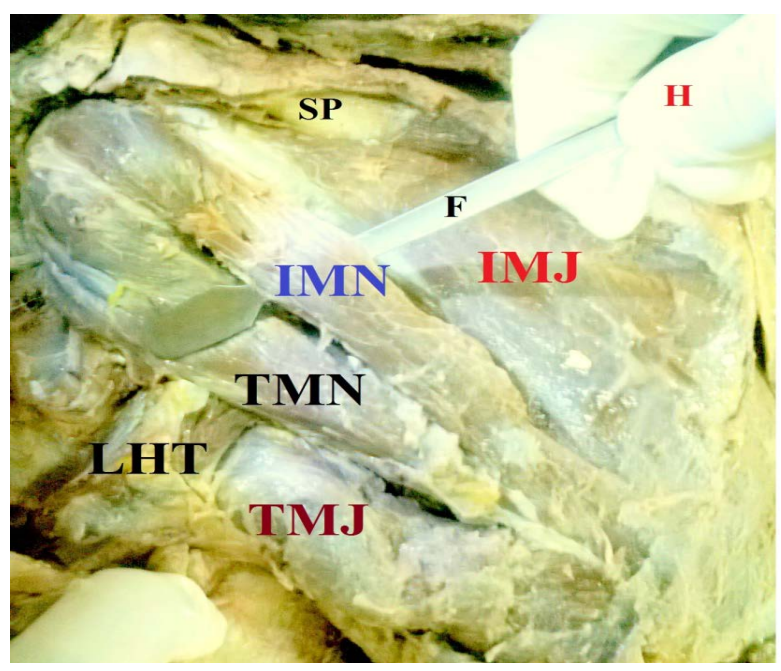

Figure 1. Photograph of the rotator cuff muscles in the posterior aspect of the left scapula and the gleno-humeral joint. SP indicates spine of scapula; IMJ indicates infraspinatus major; IMN indicates infraspinatus minor; TMN indicates teres minor; TMJ indicates teres major; LHT indicates the long head of triceps brachii muscle; H indicates the hand of the prosector; F indicates the forceps; DT-Dissecting tool. 


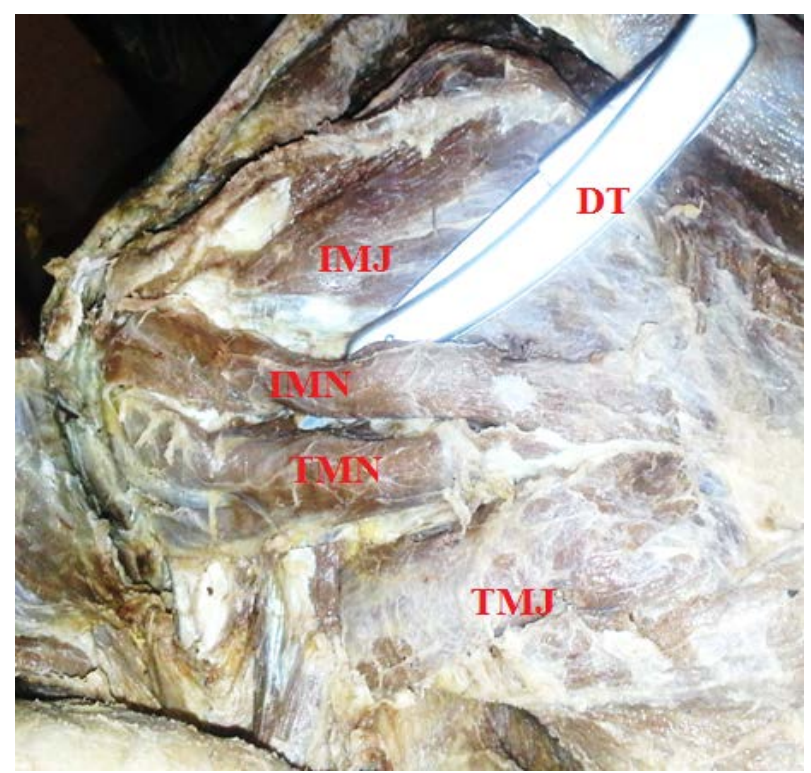

Figure 2. Image of the rotator cuff muscles in the posterior aspect of the left scapula and the gleno-humeral joint. SP indicates spine of scapula; IMJ indicates infraspinatus major; IMN indicates infraspinatus minor; TMN indicates teres minor; TMJ indicates teres major; LHT indicates the long head of triceps brachii muscle; H indicates the hand of the prosector; F indicates the forceps; DT-Dissecting tool.

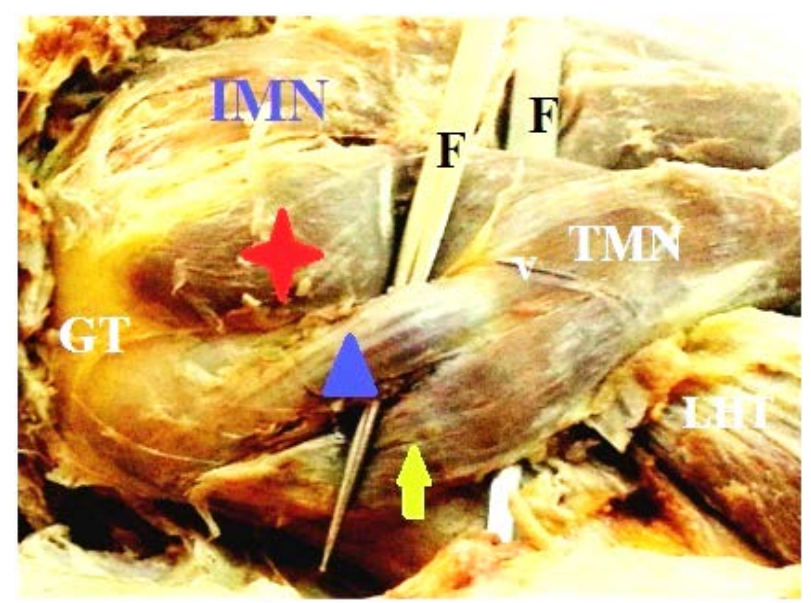

Figure 3. Picture of the postero-lateral aspect of the rotator cuff muscles of the gleno-humeral joint. IMN indicates infraspinatus minor; TMN indicates teres minor; GT indicates the greater tubercle; LHT indicates the long head of triceps; F indicates forceps. Star, arrow head and arrow indicate superior, middle and inferior tails of the teres minor respectively.

\section{Discussion}

Anatomical variations of the rotator cuff muscles are rare [2] [3]. Sometimes, the tendon of the infraspinatus may be separated from the capsule of the glenohumeral joint by a bursa [2] [3]. Infraspinatus may be fused with the teres minor [2]. Occasionally, a variant form of teres minor may occur, where teres minor splits at its termi nation into two parts and the smaller accessory part is named the teres minimus [3] [4]. A prolonged teres minor has been reported by Jain et al. [5], extending to the postero-superior and lateral portion of the capsules of the shoulder joint, over the head of humerus and further inserting on the upper part of the surgical neck of the humerus. Fraizer et al. [6] have described an accessory slip connecting the teres minor to the deltoid muscle and grossly distorting the shape of the quadrangular space. The accessory slip was innervated by the ventral rami of spinal nerves C5-6 via the posterior branch of the axillary nerve [6]. A dual blood supply from the circumflex scapular artery and the posterior circumflex artery was observed for the assessor slip [6]. 


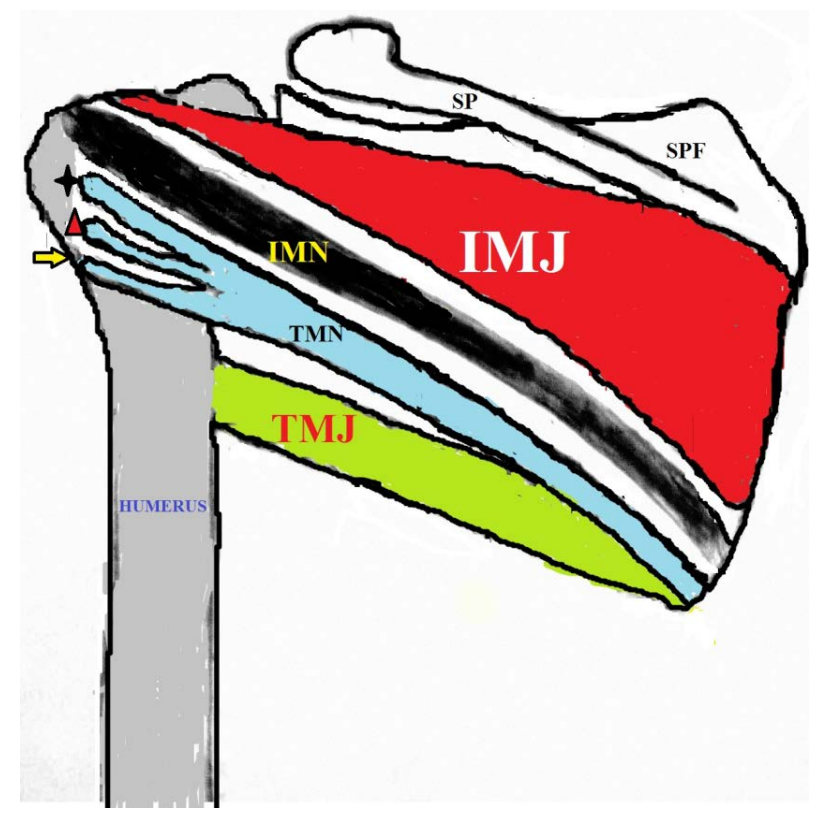

Figure 4. Schematic diagram illustrating three rotator cuff muscles in the posterior aspect of the scapula and the glenohumeral joint. SP indicates spine of scapula; SPF indicates supraspinous fossa of scapula; IMJ indicates infraspinatus major; IMN indicates infraspinatus minor; TMN indicates teres minor; TMJ indicates teres major. Star, arrow head and arrow indicate superior , middle and inferior tails of the teres minor respectively.

However, the insertion pattern of the rotator cuff muscles is quite important since it determines the opposition and steadiness of the head of humerus in the glenoid cavity checking its excessive translation [2]. The swing of humerus involves complex movements of the articular surfaces of the gleno-humeral joint which contain elements of rolling, sliding and spinning [2].

Classically, the supraspinatus, infraspinatus and teres minor typically insert on the superior, posterior and the inferior facets of the greater tubercle of humerus respectively [2] [3]. In our own case, we found double insertions on each of the facets of the greater tubercle of humerus. The superior facet receives the supraspinatus and infraspinatus major. The posterior facet receives the infraspinatus minor and superior tail of the teres minor. Also, the inferior facet receives the middle and the inferior tails of the teres minor. This, however, indicates additional attachment and reinforcement on each facet of the greater tubercle of humerus.

We therefore propose that at the shoulder joint, inferior displacement of the head of humerus is opposed by supraspinatus and infraspinatus major. Anterior displacement is prevented by infraspinatus minor and the superior tail of teres minor, while the superior displacement is prevented by middle and inferior tails. Since the infraspinatus and teres minor also participate in the lateral rotation of the gleno-humeral joint, this additional slip of muscles on each facet may predict improved ability to maneuver and develop more forceful movement at the joint. The infraspinatus major inserting on the superior facet may also assist the supraspinatus during the initial phase of abduction of the gleno-humeral joint.

The capsule of the gleno-humeral joint is also reinforced by the reported additional slips of muscles. Nonetheless, individuals possessing this kind of variations who are exposed to excessive sporting activities involving extensive movements of the gleno-humeral joint, such as javelin or shot-put throwing, may be susceptible to injuries of the additional slips.

During dissections or surgical operation of the dorso-lateral aspect of the shoulder region, it is typical to find three muscles in relation to the infraspinous region, in this supero-inferior order as infraspinatus, teres minor and teres major. These muscles are often easily identifiable, but variant muscular formation may cause confusion when examining the region. In the case we currently report, the muscles exist in this supero-inferior order: infraspinatus major, infraspinatus minor, teres minor and teres major. It requires a foreknowledge or further examination to understand such variations on sudden encounter. The infraspinatus minor could be mistaken as the teres minimus and it should be noted that the teres minimus was described as an off-shoot of the teres minor [3].

The origin of anomalous muscles may be explained on the basis of embryogenesis of muscles of the arm [7]. 
The intrinsic muscles of the upper limb differentiate in situ from the limb bud mesenchyme of the lateral plate mesoderm [7]. The upper limb buds lie opposite to the lower five cervical and upper two thoracic segments [7]. Embryologically, the teres minor and infraspinatus are developed from the dorsal muscle mass of limb bud [7]. At a certain stage of development, the muscles primordia disappear through cell death. Failure of muscle primordia to disappear during embryonic development may account for the presence of anomalous muscles slip [7].

In arthroscopy, the posterior portal is being placed between infraspinatus and teres minor muscles to avoid injury to the axillary nerve and the posterior circumflex humeral vessels [8]. It is therefore imperative for orthopaedic surgeons to understand the anatomy and/or possible variations of the posterior shoulder region. It should be noted that variant muscular slips may be surrounded by vascular structures, as we found that a branch of the posterior circumflex humeral artery lies between the superior and middle tail of the teres minor. Aberrant variations of rotator cuff muscles need to be brought to the knowledge of radiologists, surgeons and anatomists.

\section{Acknowledgements}

We sincerely appreciate the Department of Anatomy at Bowen University for all the support given towards this publication

\section{References}

[1] Moore, K.L., Dalley, A.F. and Agur, L.W.W. (2010) Clinically Oriented Anatomy. 6th Edition, Upper Limb, Philadelphia, 703-714.

[2] Salmons, S. (1995) Muscles. In: Willliams, P.L., Bannister, L.H., Berry, M.M., Patricia, C., Dyson, M., Dussek, J.E. and Ferguson, M.W.J., Eds., Gray's Anatomy, 38th Edition, Churchhill Livingstone, New York, 738-897.

[3] Bergman, R.A., Afifi, A.K. and Miyauchi, R. (2006) Illustrated Encyclopedia ot Human Anatomic Variation. (Online) Anatomy Atlases.

[4] Barry, B.P. (2008) Arthroscopy of Upper Extremity. In: Terry, C.S. and Beaty, J.H., Eds., Campbell's Operative Orthopaedics, Mosby Elsevier, Missouri, 2927-2929.

[5] Jain, M., Shukla, L. and Kaur, D. (2012) Extended Insertion of Teres Minor Muscle: A Rare Case Report. European Journal of Anatomy, 16, 224-325.

[6] Fraser, P.R., Thomas, J., Guttmann, G.D. and Rosales, A.A. (2014) Rare Accessory Slip of the Deltoid Muscle Conjoined with Teres Minor. European Journal of Anatomy, 18, 195-197.

[7] Girm, M. (1972) Ultra Structure of the Ulnar Portion of the Contrahent Muscle Layer in the Embryonic Human Hand. Folia Morphol, 20, 113-115.

[8] Andrew Jr., H.C. (2003) Surgical Techniques and Approaches. In: Terry, C.S. and Beaty, J.H., Eds., Campbell's Operative Orthopaedics, Mosby, Missouri, 93-95. 
Scientific Research Publishing (SCIRP) is one of the largest Open Access journal publishers. It is currently publishing more than 200 open access, online, peer-reviewed journals covering a wide range of academic disciplines. SCIRP serves the worldwide academic communities and contributes to the progress and application of science with its publication.

Other selected journals from SCIRP are listed as below. Submit your manuscript to us via either submit@scirp.org or Online Submission Portal.
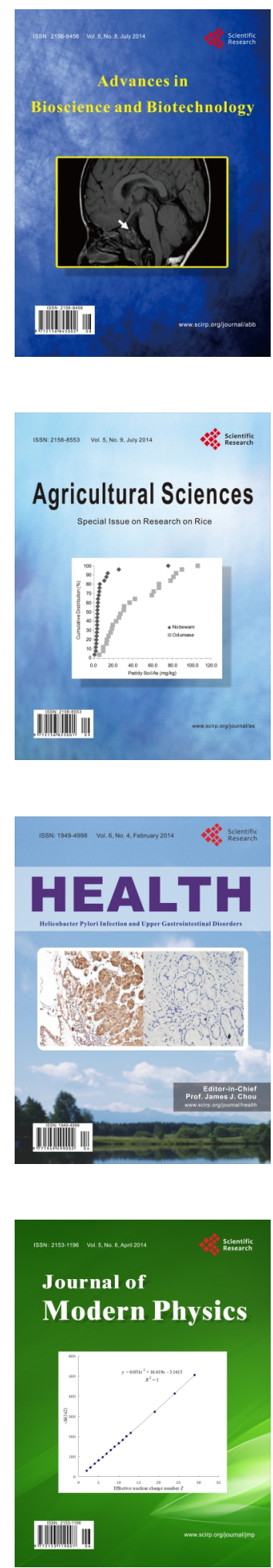
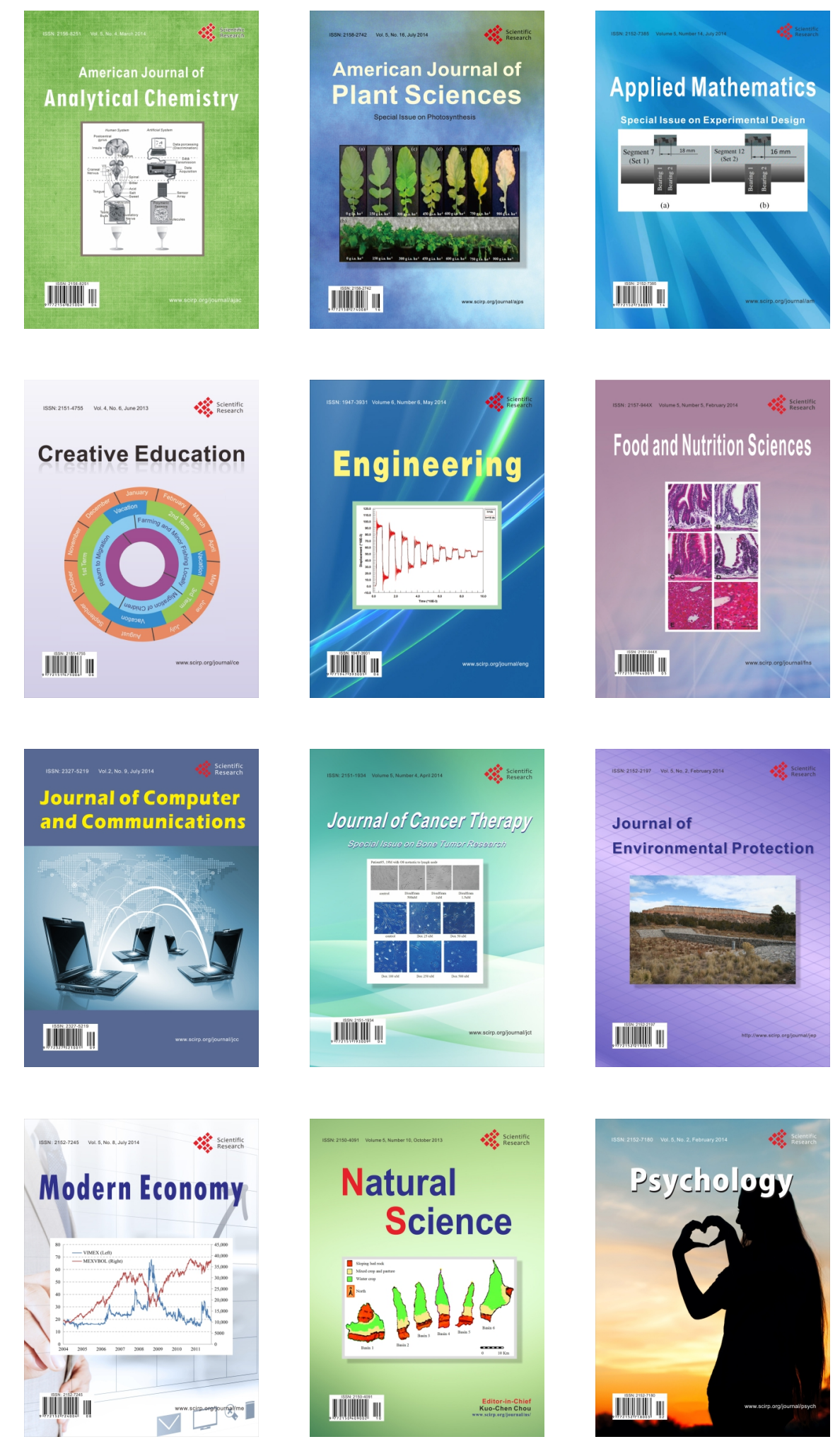